\title{
Ruga Lumina: Folding Interior Skin with Dynamic Light
}

Our human skin not only protects us, but also provides us with continuous information about our surroundings. As an interface between the body and the world, it tells us whether our surrounding is hot or cold, soft or hard, smooth or rough, sticky or creamy. The concept of 'skin' has been used as a site for rich metaphors in fashion and in architecture throughout contemporary design history. ${ }^{1}$ Interior skin, in the context of interior design, has been brought to design discourse by Lois Weinthal. An interior skin, unlike an architectural skin with its primary function as a protective envelope that mitigates the environmental impacts on our physical body, takes on a different conceptual and visual role in terms of its closer relationship with the body. ${ }^{2}$ With the advance of generative tools and digital technologies, it is now possible to envision an interior skin as a projection and extension of the body, collapsing the "subject-object and interior-exterior dichotomies and hierarchies," and connecting with "a set of intelligent and layered objects," and "a set of supple, responsive and connected subjects and interiors". ${ }^{3}$

'Ruga' is the Latin word for making wrinkles and folds. It was originally used in anatomy to refer to the folds, creases, and wrinkles found in human organs, such as human skin, and it has recently been used by material scientists to refer to the different states of folding, bending, and weaving in materials. ${ }^{4}$ In spatial design, these architectural-scaled folds, creases, and wrinkles provide malleable, adaptable and flexible spaces that allow for a body's movements in reaction to both internal and external events. In recent years, the concept of the fold has emerged as a new field of interdisciplinary research in philosophy, architecture, literature, mathematics, biology, material science, computer science, and in other fields. ${ }^{5}$ In The Fold: Leibniz and the Baroque, Gilles Deleuze uses the concept of the fold in order to explain the organization of consciousness and matter. For Deleuze, consciousness is the result of matter

that has been folded and refolded until exteriority has become interiority; "to think is to fold, to double the outside with a coextensive inside ... the general topology of thought ... now ends up in the folding of the outside into the inside ... brings the two into confrontation at the limit of living present."7 Similarly, a fold in space, instead of defining the demarcation of inside and outside, creates uncertainly between boundaries. A spatial flow from outside to inside, or from inside to outside, is therefore not fixed, but rather, in constant exchange.

Ruga Lumina, part of an ongoing research and creative project titled Ruga Interior Skin, ${ }^{8}$ is an art installation that explores the making of a smart 'skin' that collapses the subject-object and interiorexterior dichotomy and hierarchy in its responsiveness to people's movements in space through active 
luminary. Ruga Lumina's skin, like its organic name-sake, registers spatio-temporal changes and expresses active material affects; it is the in-between of subjects and objects, interiors and exteriors, selves and others, marking these dichotomies, incorporating the one in the other, but belonging fully to neither. Like and in conversation with the human beings who come to experience it, Ruga Lumina registers these dichotomous differences through the folds of its skin and its resulting expressions. Like the human body, Ruga Lumina is essentially a body that responds to the movements of other organisms and bodies within and outside of it and expresses those movements through light. It is a materialized social body of human affects.

Similar to a piece of garment that creates an intimate enclosure for the body and allows the body to both 'move with it' and be expressed by it, Ruga skin's folded topology both encloses and also registers bodies in physical and social spaces. Designed in the software Rhino/Grasshopper, topological form can be parametrically adjusted to different bodily dimensions. The parametric environment also streamlines the process from form-finding to paneling for digital fabrication. The material is polypropylene corrugation panels that are $4 \mathrm{~mm}$ in thickness. The folds allow the panels to gain significant strength while keeping flexibility at each of the folds. In each of the installations, the folded panels were connected together into strips first and then into a large skin surface. Because of the flexibility in the folds, the Ruga interior skin is essentially a movable and pliant surface. Like a piece of garment that is formed by the bodily structure, the Ruga Lumina skin is also shaped by a skeleton that is made of simple cardboard pieces and dowel rods. After the skin was stiffened by adding plastic brackets and tensioned wires hung from above, the skeleton was then removed to reveal the cavity that the Ruga skin embraced.

After setting up the Ruga skin, the process of installing projectors and the sensors began. The projectors delivered the dynamic colored light and the sensors picked up the depth information in the space. The space around Ruga Lumina is divided into six different zones on each side, and each of these zones corresponds to a set of spatial depth data and an area on the Ruga skin. When a body steps into a zone, the spatial data changes and the amount of the change is used in Process, a Java based coding language, in order to determine the hues of the colors that are used to create the gradation effects. Through the custom computer codes in Processing, the information triggers the video animations to display different colors in the areas on Ruga Lumina that correspond to the zone. The more bodies that step into a zone, then the warmer the color that will be displayed on Ruga Lumina in the area that is associated with that zone. To create the seamless integration of projected colored lights and tessellated geometry found in 
Ruga Lumina's luminous effect, the generative colored lights must be meticulously painted onto its three-dimensional topology using a mapping process. The mapping process must be accomplished on site after the physical 'skin' has been set up. Unlike a simple projection onto a flat screen, projection mapping, or video mapping, registers digital content onto the physical three-dimensional spatial construct by interacting with its surface. In other words, the digital content, acting as a virtual 'skin,' must be created based on the unique characteristics of the physical Ruga 'skin,' so that the two will be registered.

[Insert images 1-6 here]

\section{Acknowledgement:}

This project is partially supported by Indiana University's New Frontier in the Arts and Humanities Program and Indiana University's College of Arts and Humanities Institute Fellowship. The author would like to thank Kyle Overton for his work on the interactive technologies. The author would also like to thank the following individuals for their help with the installations in DCDT and 3labs: Steve Dixon, Jin Feng, Siqiao Gao, Jiang Lu, Kyle Overton, Jang Park, Karen Swanson, Dexter Wu-Corts, and Manzi Yang.

\section{Images Credits:}

The author would like to thank Kyle Overton for photographing images on image page 1 and 2 and Joseph Caputo for photographing images on image page 6.

\section{Endnotes:}

\footnotetext{
${ }^{1}$ The concept of 'skin' in design can be traced back to the mid-nineteenth century. In 1845, Charles Baudelaire, called the black frock coat "the outer skin of the modern hero," thus allowing the word skin to first take on a literal role in reference to clothing or fashion. Charles Baudelaire and P. E. Charvet, Selected Writings on Art and Artists [of] Baudelaire, Penguin Classics (Harmondsworth,: Penguin, 1972).

${ }^{2}$ Lois Weinthal, "Interior Skins," International Journal of Interior Architecture and Spatial Design, no. 2 (2013): 6471.

${ }^{3}$ W. Dubbledam, "Thing-Shapes," in Intimus: Interior Design Theory Reader, ed. M. Taylor and J. Preston (WileyAcademy, 2006), 96-100.

${ }^{4}$ M. Diab et al., "Ruga Mechanics of Creasing: From Instantaneous to Setback Creases," Royal Society A: Mathematical, Physical and Engineering Sciences 469, no. 2157 (2013): 20120753.

${ }^{5}$ Michael Friedman and Wolfgang Schäffner, On Folding : Towards a New Field of Interdisciplinary Research, Science Studies (Bielefeld: transcript, 2016), 7-29.

${ }^{6}$ For more on Deleuze's discussion on folds and human consciousness, refer to the chapter entitled "Perception in the folds" in Gilles Deleuze, The Fold : Leibniz and the Baroque, Rev. ed., Continuum Impacts (London ; New York: Continuum, 2006), 97-113.
} 
${ }^{7}$ Gille Deleuze, Faucault (Minneapolis: University of Minnesota Press, 2000), 118-9.

8 Jiangmei Wu, "Body, Form, Material, and Surface-Making of Ruga Interior Skin," Interior: Design, Architecture, Culture 8, no. 3 (2017). DOI: 10.1080/20419112.2017.1374026 